Table S1. Exclusion criteria

\title{
Exclusion criteria
}

1. Not fulfilling inclusion criteria

2. Not on a stable LLT or diet

3. Currently taking a statin other than atorvastatin, rosuvastatin, or simvastatin

4. Irregularly taking a statin or not at a registered dose

5. Use of a fibrate other than fenofibrate

6. Use of nutraceutical product or over-the-counter treatment or red yeast rice

product

7. Use of plasmapheresis

8. Recent vascular event or bariatric surgery as well as planned vascular

procedure

9. Blood pressure $>160 / 100 \mathrm{~mm} \mathrm{Hg}$

10. History of familial hypercholesterolemia

11. History of intracranial hemorrhage

12. Recent history of congestive heart failure with New York Heart Association Class III or IV

13. Newly diagnosed or poorly controlled diabetes mellitus 
14. History of loss of function of PCSK9

15. Use of systemic steroid or hormone replacement therapy unless the regimen has been stable

16. History of cancer

17. Positive for human immunodeficiency virus test

18. History of taking alirocumab

19. Hepatitis B surface antigen (+) or hepatitis C antibody (+); Beta-human chorionic gonadotropin $(+)$ or pregnancy test $(+)$; Triglyceride $>400 \mathrm{mg} / \mathrm{dL}$; Estimated glomerular filtration rate $<30 \mathrm{~mL} / \mathrm{min} / 1.73 \mathrm{~m}^{2}$; Alanine aminotransferase or aspartate transferase or creatine phosphokinase $>3 \mathrm{X}$ upper limit normal

LLT, lipid-lowering treatment; PCSK9, proprotein convertase subtilisin/kexin type 9. 
Table S2. TEAEs related to diabetes mellitus or diabetic complications (CMQ) $\dagger$, according to baseline diabetes status

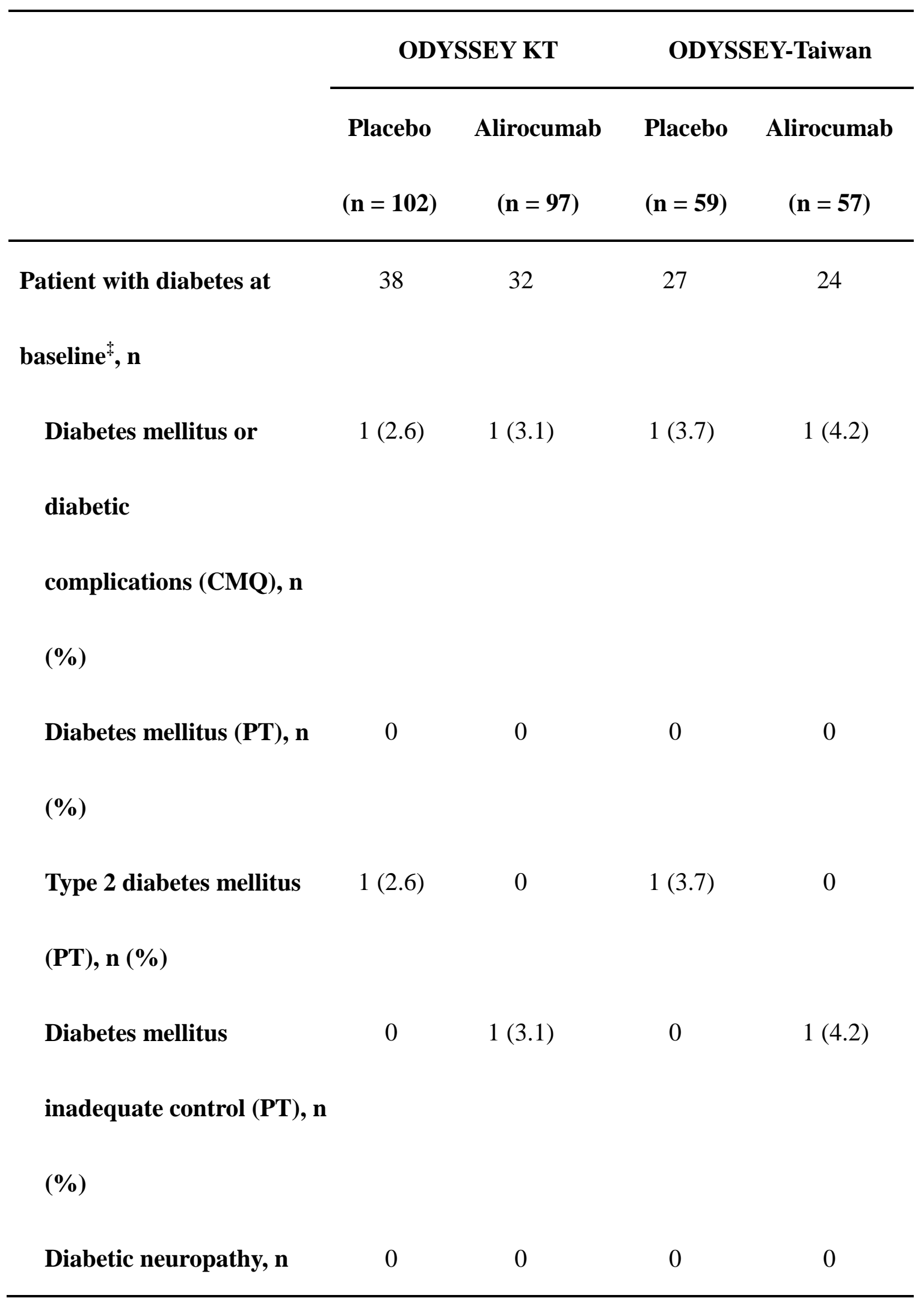


(\%)

Diabetic retinopathy, $n$

0

0

0

0

(\%)

Patient without diabetes at

64

65

32

33

baseline $^{\ddagger}, \mathbf{n}$

Diabetes mellitus or

$2(3.1) \quad 4(6.2)$

$2(6.3)$

$3(9.1)$

diabetic complications

(CMQ), n (\%)

Diabetes mellitus (PT), n

$1(1.6)$

$2(3.1)$

$1(3.1)$

$2(6.1)$

(\%)

Type 2 diabetes mellitus

$1(1.6) \quad 1(1.5)$

$1(3.1)$

$1(3.0)$

(PT), n (\%)

Blood glucose increased

0

0

0

0

(PT), n (\%)

Glycosylated hemoglobin

0

$1(1.5)$

0

0

increasing, n (\%)

Hyperglycemia (PT), n

0

0

0

0

(\%)

$\uparrow$ Selection of PTs is based on the HLGT “diabetic complications", HLT "diabetes mellitus", and HLT 
“carbohydrate tolerance analyses (including diabetes)," excluding PT "blood glucose decreased" and PT "hyperglycemia."

$\$$ According to medical history

CMQ, customized MedRA Queries; HLGT, high level group term; HLT, high level term; MedDRA, Medical Dictionary of Regulatory Activities; PT, preferred term;. 